\title{
Manifestações bucais em pacientes oncológicos
}

\author{
Winícius A. F. ARAÚJO'; Higor O. ROCHA'; Grace \\ K. M. CARNEIRO2; Natália G. GARCIA ${ }^{3}$ \\ 1 - Graduando em Odontologia na Faculdade Morgana Potrich (FAMP), Mineiros - GO, Brasil; 2 - Especialista e Mestre em Orto- \\ dontia - Professora do Curso de Odontologia da Faculdade Morgana Potrich (FAMP), Mineiros - GO, Brasil; 3 - Mestre, Doutora \\ e Pós-doutora em Ciências Odontológicas Aplicadas - Professora do Curso de Odontologia do Centro Universitário de Lavras \\ (UNILAVRAS), Lavras - MG, Brasil.
}

\section{Resumo}

Objetivos: Considerando a alta prevalência do câncer, o presente estudo teve como intuito identificar as principais manifestações bucais em pacientes submetidos a tratamento antineoplásico. Materiais e Métodos: A amostra foi constituída por indivíduos portadores de neoplasia maligna submetidos ao tratamento antineoplásico, quimioterapia e/ou radioterapia. Os quais responderam a um questionário, passaram por exame clínico e receberam orientações sobre o diagnóstico e o tratamento das manifestações bucais. Resultados: Foram avaliados 57 participantes com idade média de 56 anos, dentre os quais, a maioria foi submetida à cirurgia associada à radioterapia e quimioterapia e ainda não haviam finalizado. No exame clínico foi identificado que $91,3 \%$ dos pacientes apresentaram pelo menos uma manifestação bucal decorrente do tratamento antineoplásico, sendo a xerostomia $(21,0 \%)$ e a mucosite $(12,3 \%)$ as manifestações de maior incidência durante a realização do tratamento. Além disso, apenas $14,0 \%$ dos participantes relataram terem tido acompanhamento odontológico durante o tratamento oncológico. Conclusões: Diante da incidência relevante das manifestações bucais identificadas nos participantes deste estudo, sugere-se que a participação do cirurgião-dentista durante e após a realização do tratamento antineoplásico é imprescindível.

PALAVRAS-CHAVE: Câncer; Quimioterapia; Radioterapia; Manifestações Bucais.

AUTOR PARA CORRESPONDÊNCIA

Natália Galvão Garcia

Centro Universitário de Lavras (UNILAVRAS)

Rua Padre Jose Poggel, 506, Lavras-MG, CEP.: 3720.000 Brazil.

Telefone: +55 31 99403-3999 · Email: natggalvao@hotmail.com / nataliagalvao@unilavras.edu.br 


\section{Introdução}

Segundo o último levantamento feito pelo Instituto Nacional de Câncer José Alencar Gomes da Silva (INCA) estimou-se a ocorrência de 600 mil novos casos de câncer no país no último ano ${ }^{1}$.

Diante desses altos números, o câncer tem sido considerado uma doença avassaladora que vem incidindo sobre a sociedade, causando debilidades de estrutura física e mental do indivíduo, levando muitos a óbito ${ }^{2,3}$.

Dentre os tratamentos existentes, a quimioterapia e a radioterapia são os mais comumente utilizados. A quimioterapia se caracteriza como um método agressivo e de efeito colateral bastante evidente. $\mathrm{Na}$ qual, medicamentos são administrados de forma endovenosa ou via oral. No entanto, a ação dos medicamentos não possui seletividade apenas sobre as células tumorais, acometendo também células sadias ${ }^{4-6}$. A radioterapia se caracteriza pela exposição do paciente à radiação ionizante, a qual também pode ser bastante nociva ao paciente ${ }^{7}$. $\mathrm{O}$ método empregado sobre cada caso varia de acordo com o estágio, a gravidade e a localização do tumor. Podendo lançar mão de apenas um método isolado, ou da associação de $\operatorname{ambos}^{4-8}$.

Essa terapia antineoplásica gera imunossupressão e alterações na cavidade bucal, que muitas vezes pioram o quadro geral dos pacientes $^{9-12}$. As lesões na cavidade bucal compreendem as mais frequentes complicações oriundas desse tipo de tratamento, devido à alta sensibilidade dos tecidos e das estruturas bucais aos efeitos tóxicos da quimio e/ou radioterapia ${ }^{2,9,10}$.

Dentre as manifestações bucais que podem ocorrer, as mais comuns são mucosite, xerostomia, cáries de radiação, osteorradionecrose, candidíase, entre outras infecções bacterianas e viróticas $^{3,13-18}$.

Sendo assim, considerando a alta prevalência do câncer, o presente estudo teve como intuito identificar as principais manifestações bucais em pacientes submetidos a tratamento 
antineoplásico, quimioterapia e/ou radioterapia. E ainda orientá-los sobre o tratamento odontológico adequado, buscando dessa forma, melhorar a sua qualidade de vida.

\section{Materiais e métodos}

Este estudo foi submetido à apreciação do Comitê de Ética em Pesquisa em Seres Humanos do Centro Universitário de Santa Fé do Sul (UNIFUNEC), e aprovado pelo mesmo (CAAE: 09371919.1.0000.5428).

A amostra foi constituída por indivíduos que preencheram os seguintes critérios de inclusão: ser ou ter sido portador de uma neoplasia maligna em qualquer região do corpo, estar sendo ou ter sido submetido à quimioterapia e/ou radioterapia, aceitar participar do estudo por meio da assinatura do termo de consentimento livre e esclarecido (TCLE). Foram excluídos os indivíduos que foram tratados apenas com excisão cirúrgica, considerando que não foram avaliados apenas tumores de cabeça e pescoço, e os que se negaram a participar do estudo.

Para a coleta dos dados, os participantes foram convidados a responderem um questionário e em seguida foram avaliados por meio de exame clínico. O questionário utilizado foi auto aplicado em local reservado e sem conter informações que identificasse os participantes, levando em torno de 20 a 30 minutos. Sendo levantados dados como: idade, sexo, localização do tumor primário, tipo de tratamento, medicamentos utilizados, doenças sistêmicas concomitantes, hábitos, sintomatologia e presença de manifestações bucais durante ou após o tratamento.

O exame clínico intra e extrabucal foi realizado em todos os participantes em cadeira odontológica, sob iluminação artificial, utilizando um abaixador de língua estéril para afastar os tecidos moles bucais e, quando necessário, compressas de gaze. Foram avaliados os linfonodos, a condição de higiene bucal, presença de cárie e manifestações bucais como xerostomia, candidíase, mucosite, afta, osteorradionecrose, entre outras. 
Além disso, os participantes receberam orientações e um folder, sobre o tratamento das manifestações bucais e sobre a importância da higienização bucal adequada.

As respostas coletadas nos questionários foram tabuladas em uma planilha do Microsoft Excel. As variáveis quantitativas foram submetidas à estatística descritiva e os resultados foram expressos na forma de tabelas e gráficos com a distribuição de frequência em números absolutos e porcentuais.

\section{Resultados}

O presente estudo foi constituído por 57 participantes, sendo $34(59,7 \%)$ do sexo feminino e $23(40,3 \%)$ do sexo masculino com idade média de 56 anos (32 mínima e 80 máxima). Em relação ao tratamento a maior parte dos participantes $22(38,6 \%)$ foram submetidos à cirurgia associada à radioterapia e quimioterapia e a maioria $39(68,4 \%)$ ainda não havia finalizado o tratamento, como pode ser observado na Tabela 1.

Quanto à localização do tumor, a de maior incidência no presente estudo foi à mama (32\%), seguida por cabeça e pescoço (21\%), próstata (10\%), entre outros como apresentado pela Figura 1.

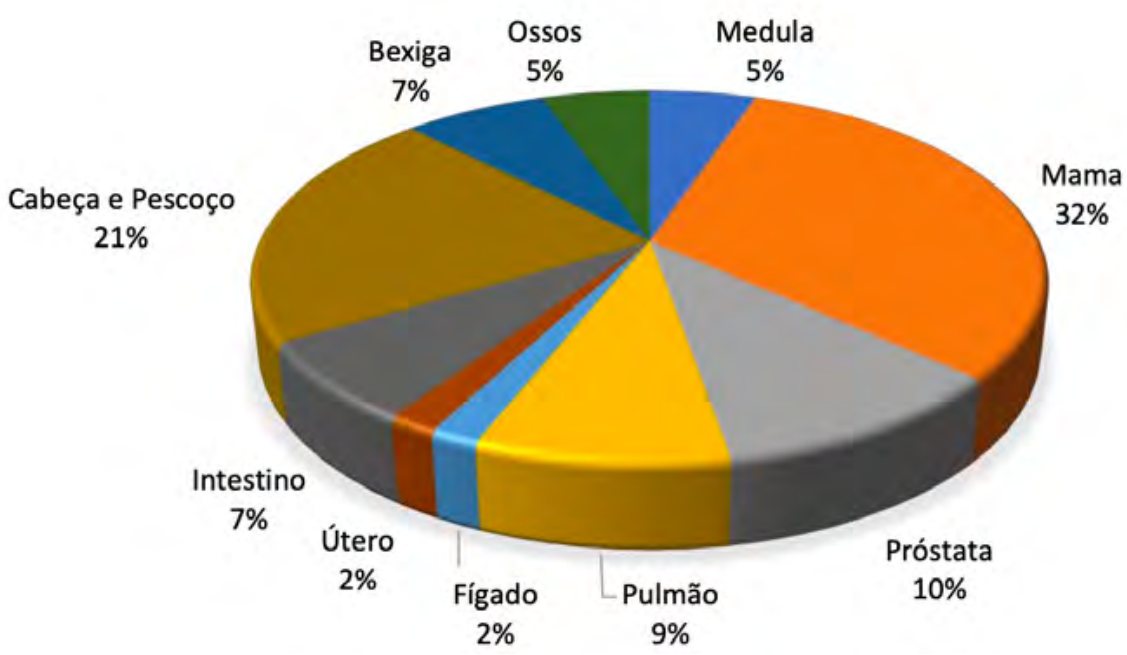

FIGURA 1 - Distribuições dos pacientes segundo tipo de neoplasia maligna. 
TABELA 1 - Perfil dos participantes segundo a idade, sexo, tempo da finalização do tratamento e tipos de tratamento realizados

\begin{tabular}{|c|c|}
\hline Características & $N(\%)$ \\
\hline \multicolumn{2}{|l|}{ Sexo } \\
\hline Feminino & $34(59,7 \%)$ \\
\hline Masculino & $23(40,3 \%)$ \\
\hline \multicolumn{2}{|l|}{ Idade Média } \\
\hline$<56$ anos & $28(49,2 \%)$ \\
\hline$>56$ anos & $29(50,8 \%)$ \\
\hline \multicolumn{2}{|l|}{ Tratamento finalizado } \\
\hline Não & $39(68,4 \%)$ \\
\hline Há menos de 1 ano & - \\
\hline Há mais de 1 ano & - \\
\hline Entre 2 a 5 anos & $8(14,0 \%)$ \\
\hline Mais de 5 anos & $10(17,6 \%)$ \\
\hline \multicolumn{2}{|l|}{ Tipo de Tratamento } \\
\hline Cirurgia + Quimioterapia + Radioterapia & $22(38,6 \%)$ \\
\hline Cirurgia + Quimioterapia & $9(15,8 \%)$ \\
\hline Cirurgia + Radioterapia & $7(12,2 \%)$ \\
\hline Quimioterapia + Radioterapia & $9(15,8 \%)$ \\
\hline Quimioterapia & $6(10,5 \%)$ \\
\hline Radioterapia & $4(7,1 \%)$ \\
\hline Total & $57(100 \%)$ \\
\hline
\end{tabular}

Fonte: Autoria própria (2020)

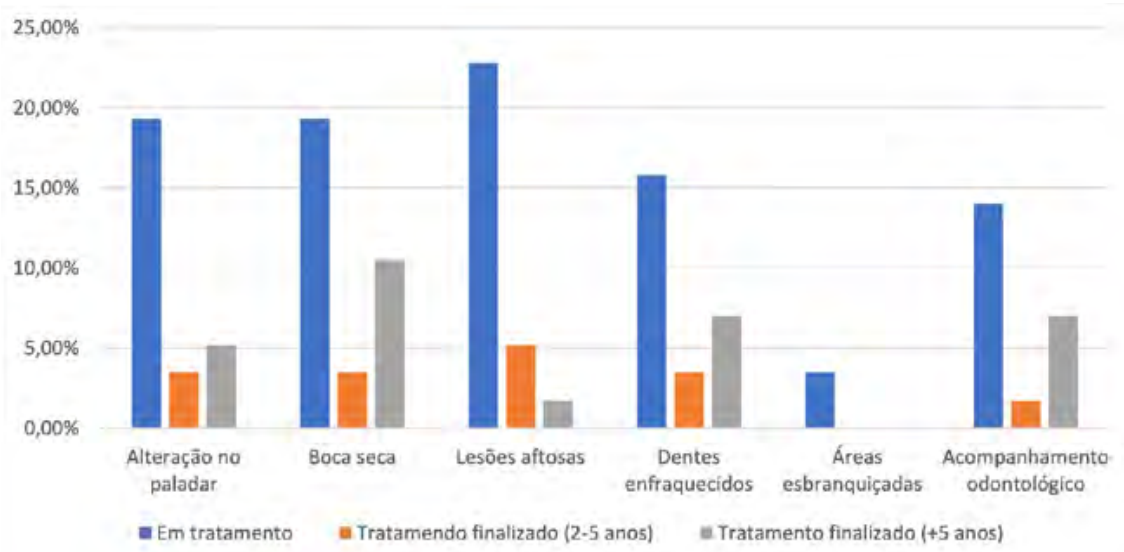

FIGURA 2 - Alterações bucais presentes durante ou após o tratamento, segundo o questionário. 
Com base nas informações obtidas por meio do questionário aplicado, ao serem indagados quanto às manifestações bucais que ocorreram durante ou depois de finalizado o tratamento, foi observada maior incidência de boca seca (33,3\%), seguida por lesões aftosas $(29,7 \%)$, alterações no paladar $(28,0 \%)$, dentes enfraquecidos $(26,3 \%)$ e áreas esbranquiçadas (3,5\%). Também foi notado que dentre as manifestações bucais relatadas pelos participantes, a mais prevalente durante o tratamento foi lesão aftosa $(22,8 \%)$ e depois de finalizado foi a sensação de boca seca (14,0\%). Além disso, apenas $14,0 \%$ dos participantes relataram terem tido acompanhamento odontológico durante o tratamento oncológico (Figura 2).

Por meio do exame clínico realizado foi possível identificar que 91,3\% dos pacientes apresentaram pelo menos uma manifestação bucal decorrente do tratamento oncológico. Sendo a xerostomia $(21,0 \%)$ e a mucosite $(12,3 \%)$ as manifestações de maior incidência durante a realização do tratamento. No entanto, nos participantes que haviam finalizado o tratamento entre 2-5 anos, a prevalência de xerostomia e mucosite foram iguais. Já nos pacientes que haviam finalizado o tratamento há mais de 5 anos, foi identificada apenas xerostomia $(8,8 \%)$ e nenhuma outra manifestação bucal, como pode ser observado na Figura 3.

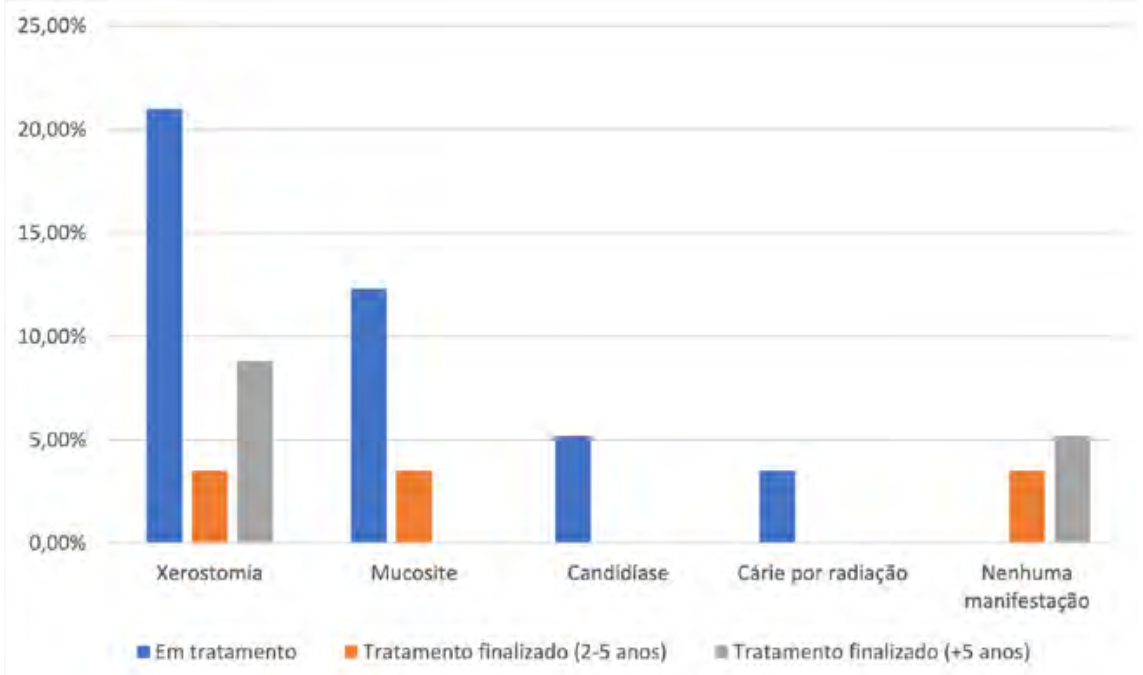

FIGURA 3 • Distribuições das manifestações bucais segundo o exame clínico. 
Vale ressaltar que foi observada uma correlação de maior ocorrência das manifestações bucais quando o paciente foi submetido à quimioterapia associada à radioterapia quando comparado com os indivíduos que foram submetidos à apenas uma dessas terapias.

\section{Discussão}

O fato da terapia antineoplásica não ser capaz de destruir as células tumorais sem lesionar células normais gera imunossupressão e provoca também o desenvolvimento de manifestações bucais agudas ou tardias, devido à alta sensibilidade dos tecidos e estruturas da cavidade bucal aos efeitos tóxicos da quimioterapia e radioterapia ${ }^{9-12,17}$.

Estudos recentes têm mostrado que a magnitude dessas manifestações bucais depende de uma série de fatores relacionados ao paciente, ao tumor e ao tratamento. Como por exemplo, a idade do paciente, localização e grau de malignidade do tumor, drogas utilizadas e tempo de duração do tratamento ${ }^{4,13-18}$. A magnitude das manifestações bucais pode agravar o quadro geral do paciente, ocasionando interrupção do tratamento e alta taxa de morbidade ${ }^{2-4}$.

No presente estudo a idade média dos pacientes foi 56 anos, os locais mais acometidos foram mama e região de cabeça e pescoço, e na maior parte dos casos, os participantes foram submetidos à cirurgia associada à radioterapia e quimioterapia.

A radiação ionizante da radioterapia e as drogas utilizadas na quimioterapia alteram o mecanismo das células normais da cavidade bucal provocando danos irreversíveis ${ }^{17}$. Lopes et al. ${ }^{13}$ (2012) avaliaram as manifestações bucais em pacientes pediátricos em tratamento quimioterápico e observaram que $83,3 \%$ dos pacientes apresentaram manifestação bucal. No presente estudo também foi diagnosticada pelo menos uma manifestação bucal em $91,3 \%$ dos pacientes participantes. 
De acordo com a literatura, dentre as manifestações bucais mais comumente encontradas estão, mucosite, xerostomia, alteração no paladar, cáries de radiação, infecções oportunistas, entre outras ${ }^{4,13-18}$. Alguns autores afirmam que a mucosite é a manifestação bucal de maior incidência, seguida pela xerosto$\mathrm{mia}^{4,13}$. Entretanto, outros apontam a xerostomia como sendo a manifestação de maior prevalência, seguida pela mucosite $e^{6,18}$. Corroborando com esses autores, neste estudo a xerostomia estava presente em $21,0 \%$ dos participantes e a mucosite em $12,3 \%$, durante a realização do tratamento. No entanto, nos participantes que haviam finalizado o tratamento entre 2-5 anos, a prevalência da xerostomia e mucosite foram iguais. E nos pacientes que haviam finalizado o tratamento há mais de 5 anos, foi identificada apenas a ocorrência da xerostomia $(8,8 \%)$.

Esses dados também foram reafirmados por meio do questionário aplicado. No qual, os pacientes relataram sensação de boca seca em 33,3\% dos casos e presença de lesões aftosas em 29,7\%. No entanto, a manifestação mais prevalente durante o tratamento foi lesão aftosa $(22,8 \%)$ e após finalizado foi a sensação de boca seca $(14,0 \%)$.

Essa maior ocorrência de manifestações como as mucosites e/ ou lesões aftosas durante a realização do tratamento pode ser justificada pela maior intensidade da reação inflamatória, ulceração e descamação da mucosa bucal provocadas pelos agentes terapêuticos nesse período ${ }^{4,17}$.

Já a xerostomia se mantém em alta incidência mesmo após finalizado o tratamento, pois, além do decréscimo transitório na produção de saliva, alguns agentes terapêuticos são capazes de causar danos irreversíveis dos ácinos glandulares e consequentemente, severa disfunção salivar de forma permanente ${ }^{17}$.

Em incidências menos significativas outras manifestações bucais como a candidíase, cárie de radiação, alteração no paladar e odontalgias também têm sido diagnosticadas ${ }^{4,13-18}$. 
Segundo Lopes et al. ${ }^{13}$ (2012) além da xerostomia e mucosite, foi observada ainda, candidíase em $41,6 \%$ dos pacientes. Hespanhol et al. ${ }^{4}$ (2010) também relataram ter encontrado essa manifestação bucal, no entanto, em uma proporção bem menos significativa (3,0\%), assim como foi observada neste estudo (5,2\%). Essa variação quanto à incidência pode está relacionada ao tipo de tumor, gênero e idade média dos pacientes participantes.

Além disso, Lopes et al. ${ }^{13}$ (2012) também afirmaram ter observado alteração no paladar em $45,8 \%$ dos casos e odontalgia em $12,5 \%$, além de outras manifestações menos relevantes. No presente estudo $28,0 \%$ dos participantes relataram alteração no paladar e apesar de não ter sido investigada a ocorrência de odontalgia, 26,3\% dos pacientes afirmaram ter notado enfraquecimento dos dentes. A alteração do paladar pode ser originada de danos nos receptores olfativos causados pela ação direta de agentes terapêuticos. Assim como também, pode ter sua origem relacionada a outras manifestações bucais, como a xerostomia e processos infecciosos. As quais também atuam favorecendo o enfraquecimento dos dentes.

Apesar da grande incidência das manifestações bucais durante e após realizado o tratamento antineoplásico, o número de pacientes que recebem suporte odontológico ainda é considerado pouco significativo, como pode ser observado neste estudo, onde apenas $14,0 \%$ dos participantes relataram terem tido acompanhamento odontológico durante o tratamento.

\section{Conclusão}

O tratamento antineoplásico pode causar manifestações bucais que afetam diretamente a qualidade de vida do paciente e consequentemente, o prognóstico do tratamento. No entanto, o número de pacientes que recebem suporte odontológico ainda é considerado pouco significativo. Sendo assim, diante da incidência relevante das manifestações bucais identificadas nos participantes deste estudo, sugere-se que a participação do cirurgião-dentista durante e após a realização do tratamento oncológico é imprescindível. 


\section{Referências}

1 - Brasil. Ministério da Saúde. Instituto Nacional do Câncer. Estimativa da incidência e mortalidade por câncer no Brasil. Rio de Janeiro: Instituto Nacional do Cânce; 2020.

2- Hancock PJ, Epstein JB, Sadler GR. Oral and dental management related to radiation therapy for head and neck cancer. J. Can. Dent. Assoc. 2003; 69(9): 585-90.

3 - Hong CHL, Hu S, Haverman T, et al. A systematic review of dental disease management in cancer patients. Support. Care Cancer. 2018; 26(1): 155-174.

4 - Hespanhol FL, Tinoco EMB, Teixeira HGC, Falabella MEV, Assis NMSP. Buccal manifestations in patients submitted to chemotherapy. Ciênc. Saúde Colet. 2010; 15(Suppl 1): 1085-94.

5 - Nascimento PBL, Santos LCO, Carvalho CN, Alves CAL, Lima SM, Cabral MMS. Avaliação das Manifestações Orais em Crianças e Adolescentes Internos em um Hospital Submetidos à Terapia Antineoplásica. Pesqui. Bras. Odontopediatria Clín. Integr. 2013; 13(3): 279-85.

6 - Araujo TLC, Mesquita LKM, Vitorino RM, Macedo AKMN, Amaral RC, Silva TF. Manifestações bucais em pacientes submetidos a tratamento quimioterápico. Rev. Cuba. Estomatol. 2015; 52(4): 10851094.

7 - Dib LL, Gonçalves RCC, Kowalski LP, Salvajoli JV. Abordagem multidisciplinar das complicações orais da radioterapia. Rev. Assoc. Paul. Cir. Dent. 2000; 54(5): 391-96.

8 - Rosso MLP, Neves MD, Araújo PF, Ceretta LB, Simões PW, Sônego FGF, Pires PDS. Análise da Condição Bucal de pacientes pediátricos e adolescentes portadores de neoplasias na Instituição Casa Guido na cidade de Criciúma (SC). Rev. Odontol. Univ. Cid. Sao Paulo. 2015; 27(3): 210-9.

9 - Ribas MO, Araújo MR. Manifestações estomatológicas em pacientes portadores de leucemia. Rev. Clín. Pesq. Odontol. 2004; 1(1): 35-41.

10 - Bellm LA, Epstein JB, Rose-Ped A, Martin P, Fuchs HJ. Patient reports of complications of bone marrow transplantation. Support. Care Cancer. 2000; 8(31): 33-99.

11 - Sonis ST, Clark J. Prevention and management of oral mucositis induced by antineoplastic therapy. Oncology. 1991; 5(12): 11-18.

12 - Santos PSS, Fernandes KS. Complicações bucais da quimioterapia. [online] 2006 [acessado em 20 janeiro 2007]. Disponível em: http://www. abrale.org.br/profissional/artigos/complicacoes_bucais.php

13 - Lopes IA, Nogueira DN, Lopes IA. Manifestações Orais Decorrentes da Quimioterapia em Crianças. Pesq Bras Odontoped Clin Integr. 2012; 12(1): 113-19. 
14 - Araujo TL, Mesquita LKM, Vitorino RM, Macedo AKMN, Amaral RC, Silva TF. Manifestações bucais em pacientes submetidos a tratamento quimioterápico. Rev. Cub. Estomato. 2015; 52(4): 15- 23.

15 - Floriano DF, Ribeiro PFA, Maragno AC, Rossi K, Simões PWTA. Complicações Orais em Pacientes Tratados com Radioterapia ou Quimioterapia em um Hospital de Santa Catarina. Rev. Odontol. Univ. Cid. São Paulo. 2017; 29(3): 230-6.

16 - Venkatesh C, Jani M, Shah A. Oral complications of chemotherapy: a review. J. Adv. Med. Dent. Scie. Res. 2015; 3(6): 99-101.

17 - Paiva MDEB, Biase RCCG, Moraes ARAP. Repercussões orais de drogas antineoplásicas: uma revisão de literatura. RFO. 2016; 21(1): 130135.

18 - Freire AAS, Honorato PSM, Macedo SB, Araújo CS. Manifestações bucais em pacientes submetidos a tratamento quimioterápico no Hospital de Câncer do Acre. J Amazon Health Sci. 2016; 2(1): 1-21. 


\title{
Oral manifestations in oncological patients
}

\begin{abstract}
Objectives: Considering the high prevalence of cancer, this study aims to identify the main oral manifestations in patients undergoing antineoplastic treatment. Materials and Methods: The sample consisted of individuals with malignant neoplasia submitted to antineoplastic treatment, chemotherapy and/or radiotherapy. They answered a questionnaire, underwent clinical examination and received guidance on the diagnosis and treatment of oral manifestations. Results: Fifty-seven participants with a mean age of 56 years were evaluated. Most of them underwent surgery associated with radiotherapy and chemotherapy and had not yet finished. In the clinical examination it was identified that $91.3 \%$ of the patients had at least one oral manifestation due to antineoplastic treatment. Xerostomia (21.0\%) and mucositis (12.3\%) were the most frequent manifestations during the treatment. In addition, only $14.0 \%$ of participants reported having had dental follow-up during cancer treatment. Conclusions: Given the relevant incidence of oral manifestations identified in the participants of this study, it is suggested that the participation of the dentist during and after antineoplastic treatment is essential
\end{abstract}

KEYWORDS: Cancer; Chemotherapy, Radiotherapy; Oral manifestations.

\section{Como citar este artigo}

Araújo WAF, Rocha HO, Carneiro GKM, Garcia NG. Manifestações bucais em pacientes oncológicos. Rev Odontol Bras Central 2021; 30(89): 85-96. DOI: 10.36065/robrac.v30i89.1452 